\title{
Un caso de tutoría grupal: aplicación de técnicas de aprendizaje cooperativo orientado a resolución de problemas de la especialidad en ingeniería ambiental
}

A case of group tutoring: Application of cooperative learning techniques aimed at solving problems at the environmental engineering degree

\author{
María Paz Araya Cabrera \\ mariapaz.araya@uv.cl \\ Héctor Andrés Andrade Caroca \\ hector.andrade@uv.cl \\ Joao Ricardo Cerqueira Pinto \\ Joao.cerqueira@uv.cl \\ Universidad de Valparaíso, Chile
}

Resumen:

En este artículo se analiza la tutoría grupal de un grupo-clase aplicando técnicas de aprendizaje cooperativo orientadas a la resolución de problemas en ingeniería ambiental, se realizará mediante la observación sistemática de clases (registro temporal de eventos y mapa de aula) en donde se evidenciará el grado de participación de los estudiantes y del docente en el aula y las interacciones de ambos. Igualmente también se aplicará una encuesta para conocer la percepción y grado de satisfacción de los estudiantes con la metodología empleada y por último se efectuará una comparación de la tasa de aprobación de la asignatura en relación al año anterior para comprobar los efectos de la metodología activa en los resultados

\section{Abstract:}

This article analyzes group tutoring for an environmental engineering group-class to which techniques of cooperative learning aimed at problem solving were applied. The analysis was undertaken through systematic observation of classes (temporary registration of events and map of classroom) where student and teacher rate of participation and interaction in the class will be measured. Likewise, a survey will be applied to know the perception and degree of satisfaction of the students with the methodology used and finally a comparison of the rate of approval of the subject in relation to the previous year will be undertaken to verify the effects of this active methodology regarding learning outcomes. The results have been satisfactory, 
Un caso de tutoría grupal: aplicación de técnicas de aprendizaje cooperativo orientado a resolución de problemas de la especialidad en ingeniería ambiental

María Paz Araya Cabrera, Héctor Andrés Andrade Caroca y Joao Ricardo Cerqueira Pinto

de aprendizaje. Los resultados han sido satisfactorios, aumenta la participación en clase aunque otros deben reconsiderarse por desconocimiento del método empleado.

\section{Palabras clave:}

Metodología; tutoría grupal; aprendizaje cooperativo; ingeniería; satisfacción. participation in class increases although other results should be reconsidered due to lack of knowledge about the method.

\section{Key words:}

Methodology; group tutoring; cooperative learning; engineering; satisfaction.

\section{Résumé:}

Dans cet article on analyse le tutorat de groupe d'un groupe-classe en utilisant des techniques d'apprentissage coopératif visant à résoudre les problèmes en ingénierie environnementale, cette étude sera effectuée par le biais de l'observation systématique des classes (journal des événements temporaires et carte classe) où il sera évident le degré de participation des étudiants et des enseignants dans la salle de classe et les interactions entre les deux. Aussi une enquête pour connaître la perception et la satisfaction des étudiants avec la méthodologie utilisée et enfin une comparaison du taux d'approbation du sujet au cours de l'année précédente doit être faite pour vérifier les effets de la méthodologie active sont également applicables aux résultats d'apprentissage. Les résultats ont été satisfaisants, la participation de la classe a augmenté tandis que d'autres devraient être reconsidérés dû à la méconnaissance de la méthode employée.

\section{Mots clés:}

Méthodologie; groupe tutorat; I'apprentissage coopératif; I'ingénierie; satisfaction.

Fecha de recepción: 24-2-2017

Fecha de aceptación: 3-5-2017

\section{Introducción}

La educación superior en Chile ha ido migrando hacia sistemas de aprendizaje centrados en los estudiantes, en donde la formación debe ir más allá de la función clásica de transmisión de conocimiento y de capacitación, reconsiderando que debe dar respuesta al desarrollo integral del estudiante, ello comporta la necesidad de formar a personas capaces de desempeñar no sólo un rol profesional, sino también un rol en la sociedad en la que conviven. Esta idea es crecientemente aceptada por organismos nacionales e internacionales y la defienden muchos analistas y autores, entre los que destacamos a Lázaro (1997), Hernández y Domínguez (1998), Martínez, Buxarrais y Esteban (2002), Rodríguez Moreno (2002), Zabalza (2003), Pino y Ricoy (2006) y González Maura y González Tirados (2007).

En las Universidades del Consejo de Rectores (CRUCH) de Chile, la tutoría está dirigida principalmente a estudiantes de los primeros años 
de universidad, con el objetivo de orientarles en la vida académica, para de ésta forma, mejorar los índices de retención en los primeros años de universidad; por ejemplo en la Universidad de Valparaíso se encuentra el Programa de Aprendizaje Colaborativo (PAC), en donde el docente es un tutor y estudiantes de años superiores trabajan como mentores para colaborar en comunidades de aprendizaje conformadas por estudiantes de primer año, para estudiar o solucionar problemáticas académicas.

Actualmente, la tutoría se concibe como una estrategia metodológica que contribuye en la orientación para los estudiantes tanto de los primeros años como para los estudiantes de todos otros niveles superiores, incluso para aquellos estudiantes que están a pasos de realizar su inserción profesional. En este sentido, la tutoría tiene matices del área profesional, por lo que el tutor debe ser un especialista en el área de formación (por ejemplo ingeniero, como el caso que nos ocupa), esto es importante pero no suficiente, también debe tener capacidad de mediación en el aula con sus estudiantes, debe tener empatía y capacidad de diálogo, y criterio para hacer críticas constructivas, puesto que el docente tiene la responsabilidad de entregar lineamientos profesionales claros que motiven al estudiante para lograr el éxito académico.

Ante esta realidad, la tutoría académica universitaria debe ofrecer asesoramiento y apoyo técnico a los estudiantes en sus tres ámbitos: académico, profesional y personal (Martínez Clares, P., Pérez Cusó, J. y Martínez Juárez, M., 2014). Desde esta perspectiva se contribuye a un claro desarrollo integral de las personas en su paso por la Universidad; No obstante, su enorme potencial queda a menudo sesgado debido al general desconocimiento de su función y aplicación metodológica.

En este artículo se presenta una experiencia de tutoría en una asignatura de la Carrera de Ingeniería Ambiental de la Facultad de Ingeniería de la Universidad de Valparaíso de Chile, en donde el docente es el tutor de grupos pequeños y autónomos de trabajo y plantea el uso de las tutorías académico-universitarias en sesiones bien organizadas. La finalidad o propósito de este estudio es mostrar una experiencia o buena práctica de tutoría universitaria aplicado en estudiantes de cuarto año de la asignatura de Meteorología y Contaminación Atmosférica (IAM 324). 
Un caso de tutoría grupal: aplicación de técnicas de aprendizaje cooperativo orientado a resolución de problemas de la especialidad en ingeniería ambiental

María Paz Araya Cabrera, Héctor Andrés Andrade Caroca y Joao Ricardo Cerqueira Pinto

\section{Método}

El propósito general del trabajo se concreta en los siguientes objetivos:

1. Describir como el docente implementa la tutoría en su docencia e identificar como la tutoría interviene en la participación de los estudiantes en el aula

2. Conocer la opinión que tiene el alumnado sobre la tutoría implementada en la asignatura de Meteorología y Contaminación Atmosférica y la importancia que le conceden para su desarrollo profesional

3. Valorar qué demandan los estudiantes a la tutoría y al tutor universitario.

4. Conocer y categorizar las acciones de mejora que propone el alumnado para el buen desarrollo de la tutoría universitaria

5. Identificar el impacto de la aplicación de la tutoría en los resultados académicos de los estudiantes.

El presente estudio se trata de una investigación descriptiva, ya que se detalla una realidad educativa $y$, a su vez, se miden de manera independiente los conceptos o variables a las que se refiere (Buendía, Colás \& Hernández, 1998). Se basa en un diseño no experimental y transversal con un enfoque analítico mixto. Se emplea una metodología tipo encuesta o survey.

Los participantes en este trabajo son un docente, junto a sus 23 estudiantes pertenecientes a $4^{\circ}$ año de la carrera de Ingeniería Ambiental de la Universidad de Valparaíso, Chile, que se encuentran cursando la asignatura de Meteorología y Contaminación Atmosférica (IAM 324).

La elección de ésta asignatura está motivada por diferentes factores. En primer lugar, con la intención de representar la implementación de esta metodología en un curso de semestre superior con estudiantes que están ad portas de realizar su primera práctica profesional y en segundo lugar, la asignatura es reconocida como compleja, con una histórica alta tasa de reprobación por lo que, es interesante para ver qué efectos puede tener la tutoría en el rendimiento de los estudiantes.

Para recoger la información se han utilizado dos instrumentos; el primero consiste en una pauta de observación de clases con un registro temporal de eventos (Mesa, V., 2010), para identificar las principales interacciones que ocurren al interior del aula entre docente-estudiante, 
estudiante - estudiante, y docente/estudiante-contenido, se considera el contexto y se observa de forma directa la implementación de la metodología por parte del docente y sus efectos en la participación de los estudiantes en el aula. A partir de esa identificación, entre otros datos, se puede medir la calidad de las interacciones que ocurren en la clase. Se registra la hora en que se observa la acción, el dialogo que se genera y cuando se usa la pizarra u otro medio. El momento en que se toman notas depende del observador (cada 5 minutos, cada vez que haya una interacción, etc.) depende de lo que quiera observar o a lo que le quiera dar énfasis. A continuación se presenta el formato de la tabla para el registro temporal de eventos en el aula:

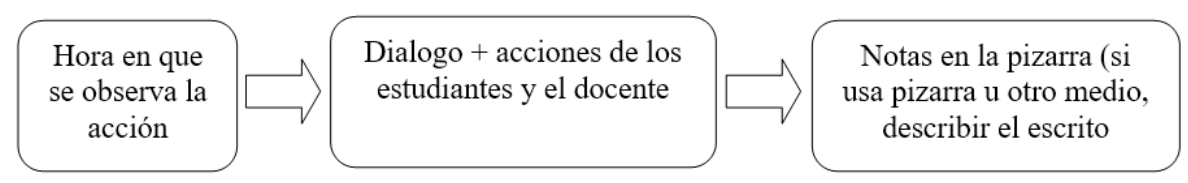

Figura 1. Formato de registro temporal de eventos.

Éste análisis observacional y descriptivo se continúa con un segundo instrumento: "Mapa de registro de interacciones y eventos en el aula" (Mesa, V., 2010), el cual permite describir gráficamente el proceso de instrucción en el aula de manera objetiva, como una fotografía, mostrando lo que efectivamente está ocurriendo en una unidad de tiempo dentro de la clase. Esto permite entregar una métrica de la participación de los estudiantes y la frecuencia de interacciones entre docente y estudiantes y entre los estudiantes (véase la figura 2): 

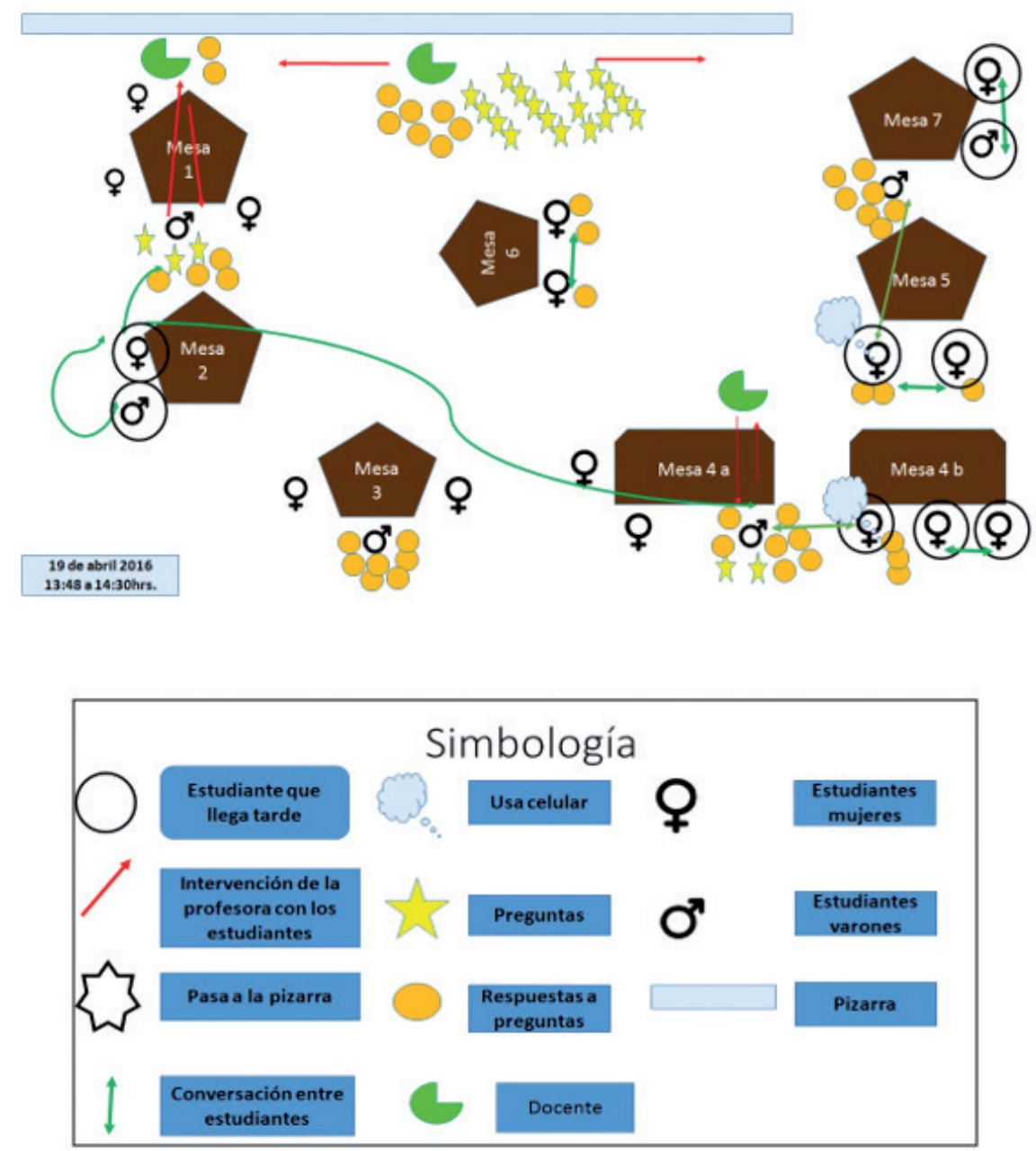

Figura 2. Ejemplo de Mapa de registro de interacciones y eventos en el aula.

Con este instrumento se puede demostrar el grado de participación, la interacción y la calidad de la interacción que ocurre en el aula; se hace a través de una simbología para representar docente, estudiante e interacciones, entre otros.

Por otra parte, para obtener información de los estudiantes se utilizó una encuesta diseñada ad-hoc denominada "Encuesta estudiante acerca de su percepción sobre la tutoría". La encuesta consta de dos Secciones; la Primera (I): Evaluación por aspecto, está dividida en 4 dimensiones con 13 afirmaciones en las que es preciso graduar la intensidad de 
la respuesta en las distintas opciones planteadas a partir de una escala tipo Likert con cinco niveles de estimación [Muy en desacuerdo-muy de acuerdo]. La segunda (II): Evaluación global, con una pregunta tipo Likert como anteriormente, dos preguntas con escala de evaluación de 1 a 7 y una pregunta abierta que recoge comentarios y/o sugerencias.

Este método permite dar respuesta a problemas tanto descriptivos como de relación de variables, a través de la formulación de una serie de cuestiones a un grupo de personas que configuran la muestra de estudio y que, por tanto, tienen la capacidad para responder a dichas preguntas, para concluir con la descripción y el establecimiento de relaciones que darán respuesta al propósito de la investigación. En el presente trabajo nos centraremos en describir y analizar aquellos ítems directamente relacionados con los objetivos propuestos.

El procedimiento seguido a lo largo de este estudio responde a las siguientes etapas: a) observación de clases utilizando la pauta del registro temporal de eventos en donde el docente está aplicando la tutoría; b) composición de los respectivos mapas de observación de interacciones en el aula; c) diseño de la encuesta por el equipo de investigación de este trabajo, seguido de un proceso de validación de contenido de tipo cualitativo a través de un juicio de expertos; d) recogida de información a través de la administración de la encuesta al termino de la segunda unidad de la asignatura en donde se implementó la tutoría, con el fin de que los alumnos hayan tenido la ocasión de experimentar diferentes momentos y situaciones relacionadas con la tutoría; e) análisis de la información, y en último lugar, f) interpretación de los resultados.

La información obtenida a partir de las cuestiones abiertas formuladas, se someten a un análisis de contenido que permite obtener la categorización de las respuestas dadas a dichas preguntas para una mejor comprensión.

\section{Resultados}

La presentación de resultados se realiza en relación a los objetivos de la investigación planteada. En cuanto al Objetivo 1 se obtienen los siguientes resultados:

Contexto de la clase: se trata de una asignatura adscrita al VI semestre de la carrera de Ingeniería Ambiental; La clase corresponde a una sesión teó- 
rico- práctico, con una actividad de taller grupal, en el que los estudiantes desarrollan un caso a través de tutoría en pequeños grupos. Es una modalidad donde los estudiantes deben dar solución a un problema en un contexto real en base a los conocimientos previos y la información entregada en clase.

El docente en las primeras dos sesiones hace la presentación de un estudio de caso y a continuación conforma los grupos de trabajo que va a tutorizar. La primera sesión es de baja participación de los estudiantes en el aula, tal y como se representa gráficamente a continuación, en los primeros 10 minutos de la sesión:

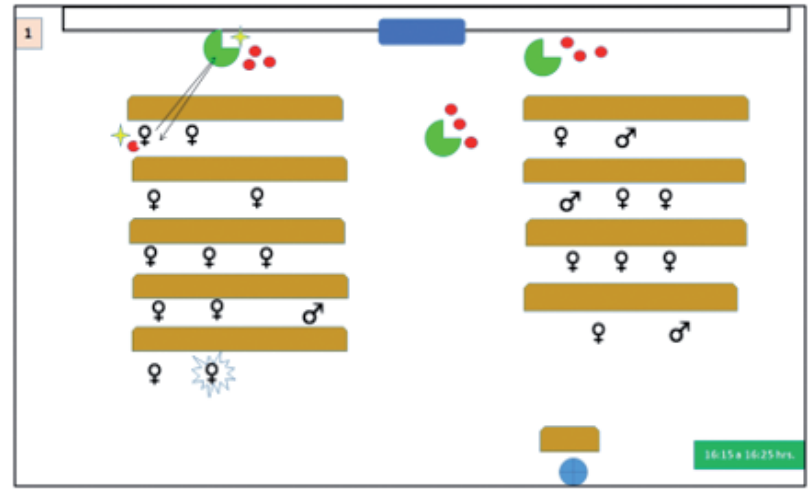

Figura 3. Representación gráfica de la clase durante los primeros 10 minutos.

Una vez que se han conformado los pequeños grupos de trabajo con dos o tres estudiantes como máximo, se puede observar en las siguientes sesiones una mayor interacción entre estudiantes y entre estudiantes y docente.

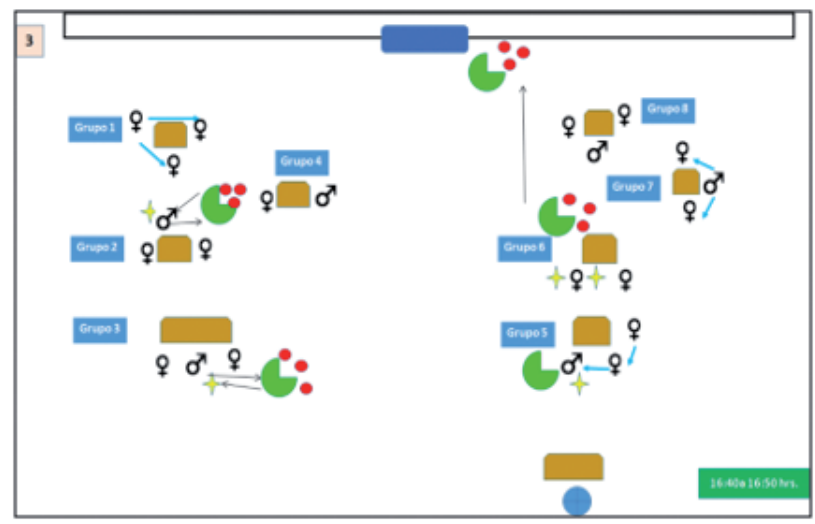

Figura 4. Representación de la clase con trabajo en pequeño grupo y tutoría. 
Cuando se realiza una sesión de tutoría a uno de los grupos, se observa la interacción entre estudiantes y docentes, sin embargo al observar el registro temporal de eventos se visualiza que quien más habla y entrega información es el docente. Se aprecia que el docente les responde las preguntas más que guiar en la resolución del problema planteado, lo que demuestra que el docente tiene carencias en la implementación de la metodología ya que tiende a entregar las respuestas como en una clase tradicional.

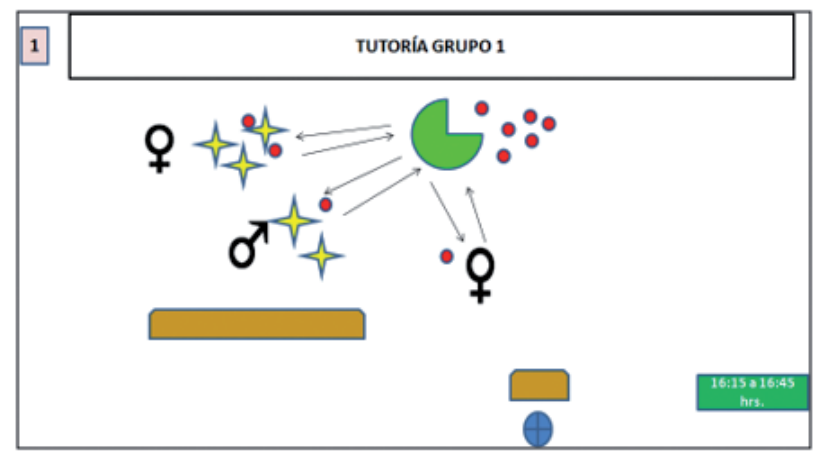

Figura 5. Representación de trabajo de tutoría.

De forma general y sintética, se puede realizar una síntesis del análisis de las clases con tutoría, teniendo en cuenta diversos aspectos:

\section{Organización de la clase}

- El docente activa conocimientos previos recordando y relacionando lo que se ha trabajado en las sesiones anteriores con la actividad a desarrollar en esta sesión.

- Los objetivos a alcanzar en las sesiones fueron claros, mostrando coherencia entre los objetivos y los contenidos asociados

- La discusión grupal es una estrategia adecuada, siempre y cuando se pueda verificar que todos los estudiantes están participando.

- Hay una secuencia lógica en el desarrollo de los contenidos

- El docente organiza a los estudiantes en pequeños grupos de dos o tres personas como máximo, lo que permite mayor participación de todos los estudiantes en clases.

- En relación al ritmo de la clase para la comprensión del estudiante, hay momentos en que el docente avanza muy rápido con conceptos técnicos complejos que no quedan del todo claro y los estudiantes no hacen preguntas o no responden, quedando la incertidumbre de si comprendieron o no.

- El docente no hace un cierre con un resumen de lo visto en la clase, quedando ambiguo el cierre de la sesión. 
Un caso de tutoría grupal: aplicación de técnicas de aprendizaje cooperativo orientado a resolución de problemas de la especialidad en ingeniería ambiental

María Paz Araya Cabrera, Héctor Andrés Andrade Caroca y Joao Ricardo Cerqueira Pinto

\section{Uso de materiales de instrucción}

- El contenido fue adecuado, riguroso y desafiante.

- El material utilizado estaba bien preparado, salvo que hubo una información errónea que los estudiantes percataron pues los confundía.

- La pizarra fue usada para la introducción de la sesión y para explicar un par de preguntas.

- El docente posee letra clara, pero atrás es poco visible; el uso de la pizarra fue un tanto desordenada, pero permite que los estudiantes a lo menos aclaren dudas. El dar la espalda mucho rato provoca que los estudiantes se distraigan mucho.

\section{Presentación del contenido}

- Buena voz con variada entonación, todos podían escuchar, pero había bastante ruido ambiente en algunas ocasiones

- El docente fue claro en las explicaciones, aunque en momentos algunos conceptos técnicos complejos no quedaron muy claros.

- Poco contacto visual, ya que el docente generalmente cuando habla o explica un tema mira hacia la pizarra o habla desde la distancia de la pizarra sin acercarse a ellos.

- Escucha atento las preguntas de los estudiantes

- Define los conceptos más difíciles, pero no todos

- Utiliza ejemplos concretos

- Para explicar procesos complejos busca distintas imágenes para lograr la comprensión de lo que intenta explicar

- Se utiliza el humor adecuadamente

- La discusión grupal es un buen método para comprometerlos con la asignatura

\section{Interacción profesor- estudiantes}

- El interrogatorio hacia los estudiantes como grupo no es muy eficaz, ya que obtiene pocas o nulas respuestas. En general él docente hizo preguntas constantemente sobre si "entendían" "quedó claro", sin embargo los estudiantes se resisten a responder a las inquietudes o a formular ellos sus propias preguntas.

- El docente responde las preguntas con amabilidad

- Llama a los estudiantes por su nombre

- Fomenta la discusión y el planteamiento de preguntas

- El docente atiende a los estudiantes que están más enganchados con el tema, aquellos que hacen preguntas, pero al resto no se les pide más participación

- Los estudiantes, unos pocos, responden con eficacia a las preguntas, en general dan respuestas breves o poco elaboradas, de mera insegura.

- El ritmo de la clase a veces fue muy rápido en relación al nivel de los estudiantes.

- El docente hace comentarios luego de las preguntas 
- Hubo algunos estudiantes que no estaban atentos a las instrucciones o estaban un poco distraídos. Algunos no entendían los temas propuestos y por ende al no preguntar hacían otras cosas (usar celular, conversar otros temas con sus compañeros, comer, etc.)

- En general el docente sigue siendo el protagonista de la clase, ya que es él quien pregunta y es él quien da respuestas.

- Falta autonomía por parte de los estudiantes demostrado en el tipo de preguntas que hacen: ¿no entendí?, ¿dónde lo encuentro?

Paralelamente se trató de identificar como la tutoría interviene en la participación de los estudiantes en el aula, para ello se realiza un análisis del compromiso de los estudiantes, llegando a las siguientes conclusiones:

- Interés de los estudiantes: en general hay un alto interés por parte de los estudiantes, lo que se refleja en la asistencia a las actividades y a las sesiones de tutoría

- Entusiasmo: si bien hay entusiasmo, éste decae cuando ven que deben trabajar de manera autónoma y no con el docente como protagonista, lo que les desconcierta y desanima en varias ocasiones, puesto que carecen de herramientas de investigación y de trabajo colaborativo.

- Participación: la participación es intencionada por el docente al hacer preguntas socráticas, los estudiantes en general son temerosos de hacer consultas o de dar su opinión, hay un sesgo con la asignatura.

- Interacción estudiante a estudiante: dentro de las actividades propuestas se genera una fuerte interacción entre los estudiantes, pero los roles dentro del grupo no quedan bien definidos, puesto que no tienen una clara organización. Al momento de aclarar dudas, generalmente ocurre que solo un integrante por grupo haga preguntas o esté atento a las respuestas u orientaciones que entrega el docente, generalmente tienden a tomar un rol pasivo en donde esperan que se le entreguen contenidos e instrucciones para seguir, falta autonomía, incluso dentro de un grupo.

En relación al Objetivo 2, se registraron un total de 21 encuestas contestadas, representando el $91 \%$ de los estudiantes de la asignatura. El análisis de las respuestas se realizó de aquellos ítems relacionados con el propósito de este estudio y en relación a la Sección I se obtienen los siguientes resultados: 
- "El método de tutoría contribuye significativamente a integrar conocimientos de asignaturas anteriores"
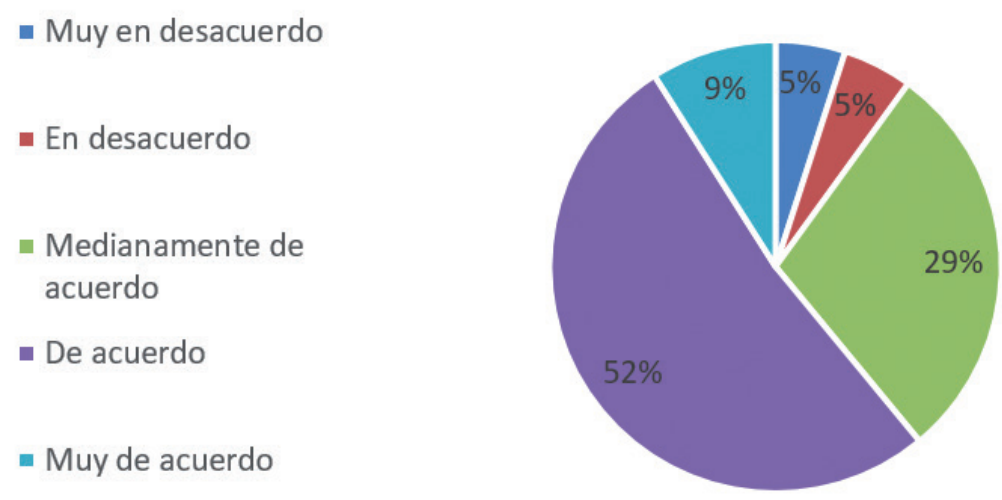

Grafico 1. Respuestas de los estudiantes en relación a la contribución de la tutoría en la integración de conocimientos anteriores.

Un 52\% manifiesta estar "de acuerdo" con la afirmación puesto que las actividades propuestas necesitan de los conocimientos adquiridos en las asignaturas anteriores. Sin embargo un 29\% revelan estar "medianamente de acuerdo" puesto que plantean que no necesariamente se recuerda todos los conceptos analizados en anteriores asignaturas y no se pueden dar por interiorizadas puesto que no se puede recordar todo.

- "El método de tutoría contribuye significativamente al logro de mis aprendizajes"
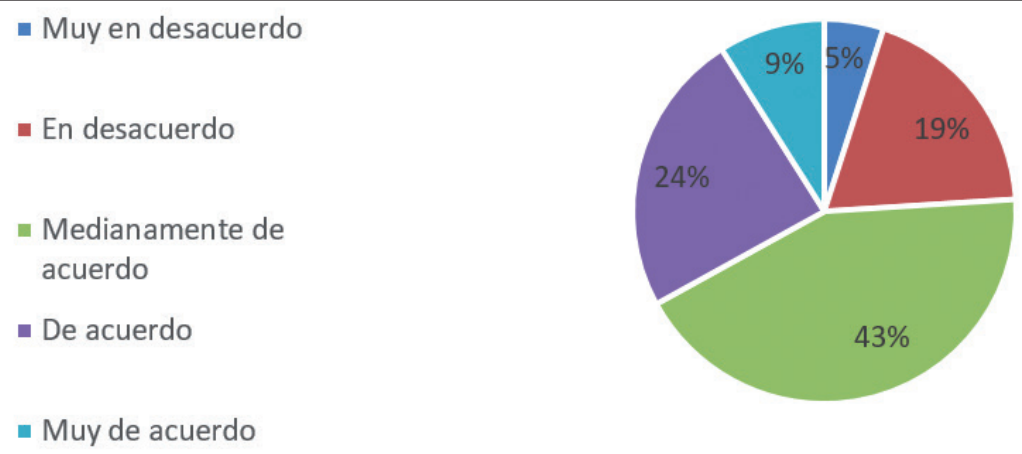

Grafico 2. Respuestas de los estudiantes en relación a la contribución de la tutoría en su aprendizaje. 
El $43 \%$ presentan estar "medianamente de acuerdo" en que esta forma de hacer clases contribuye significativamente al logro de sus aprendizajes; un 24\% manifiesta estar "de acuerdo" con la afirmación y el $19 \%$ de los participantes declaran estar "en desacuerdo", por lo que tal vez los estudiantes no tienen claridad de su rol activo en este tipo de metodologías o no entendieron el valor de desarrollar actividades formativas de este tipo, por lo que se deben buscar estrategias para que el estudiante conozca la metodología y se motive a ser más participativo en su proceso de aprendizaje, ya que insiste que es el docente quien debe entregarle la información.

- "Se ha despertado en mí el interés por investigar con mayor profundidad los temas vistos en la sesión"

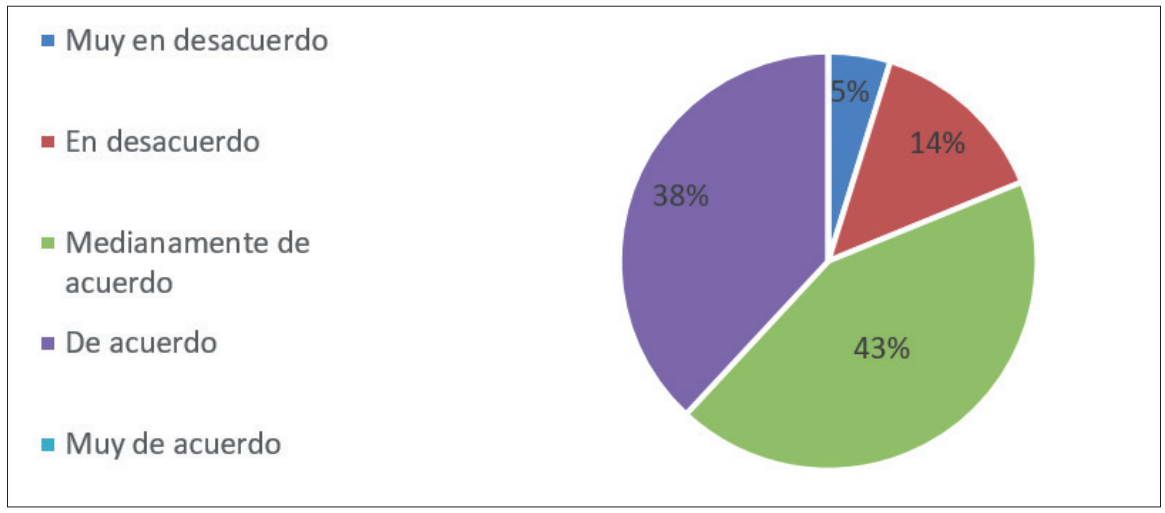

\section{Grafico 3. Respuestas de los estudiantes en relación al interés por investigar en profundidad los temas analizados en clases.}

El 43\% exhibe estar "medianamente de acuerdo" en que hay mayor interés por investigar con mayor profundidad los temas vistos en la sesión; un 38\% manifiesta estar "de acuerdo" con la afirmación y un $14 \%$ está "en desacuerdo", por lo que hay que se observa que el general de los estudiantes no tienen estrategias claras de búsqueda de información relevante y tal vez por eso no se motiven a profundizar los contenidos. 
- "Si me dan a elegir, prefiero este tipo de enseñanza"

$\begin{aligned} & \text { - } \text { - Eny en desacuerdo } \\ & \text { - Medianamente de } \\ & \text { acuerdo } \\ \text { - } & \text { De acuerdo } \\ & \text { - Muy de acuerdo }\end{aligned}$

Grafico 4. Respuestas de los estudiantes en relación a la preferencia de la metodología de tutoría.

El 33\% declara estar "medianamente de acuerdo" en que prefieren este tipo de enseñanza. Un 14\% está "de acuerdo" con la afirmación y sin embargo un $29 \%$ manifiestan estar "en desacuerdo", por lo que esto puede mostrar que algunos de los estudiantes no están muy acostumbrados a tener un rol activo dentro del aula y prefieren el rol tradicional del docente como el más activo y los estudiantes más receptivos y pasivos. Esto se puede mejorar realizando una inducción a los estudiantes y entregándoles pautas y tipos para tener éxito en las actividades propuestas.

Respecto a la Dimensión 3: Estrategias de seguimiento y evaluación, los resultados son:

- "Las actividades consideraban instancias de retroalimentación del desempeño de los estudiantes"

- Muy en desacuerdo

- En desacuerdo

- Medianamente de acuerdo

- De acuerdo

- Muy de acuerdo

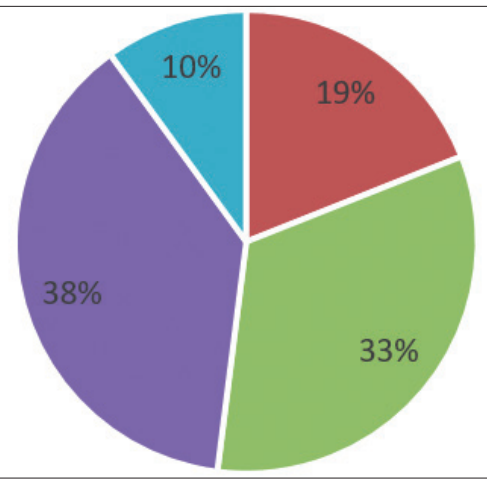

Grafico 5. Respuestas de los estudiantes en relación al desarrollo de retroalimentación dentro de la metodología. 
El 38\% expresan estar "de acuerdo" en que las actividades consideraban la retroalimentación de su desempeño; un 33\% manifiesta estar "medianamente de acuerdo", lo que indica que hay una percepción positiva respecto a la retroalimentación y un 19\% está indeciso en su respuesta al responder "en desacuerdo", por lo que hay que analizar si todos los estudiantes aclaran sus dudas o si les queda claro en que fallaron.

- "Considera adecuado el número de sesiones planificadas para el desarrollo de la tutoría (una para la presentación, cinco para aclaraciones y feedback y dos para la presentación de los resultados)"

Grafico 6. Respuestas de los estudiantes en relación al número de sesiones

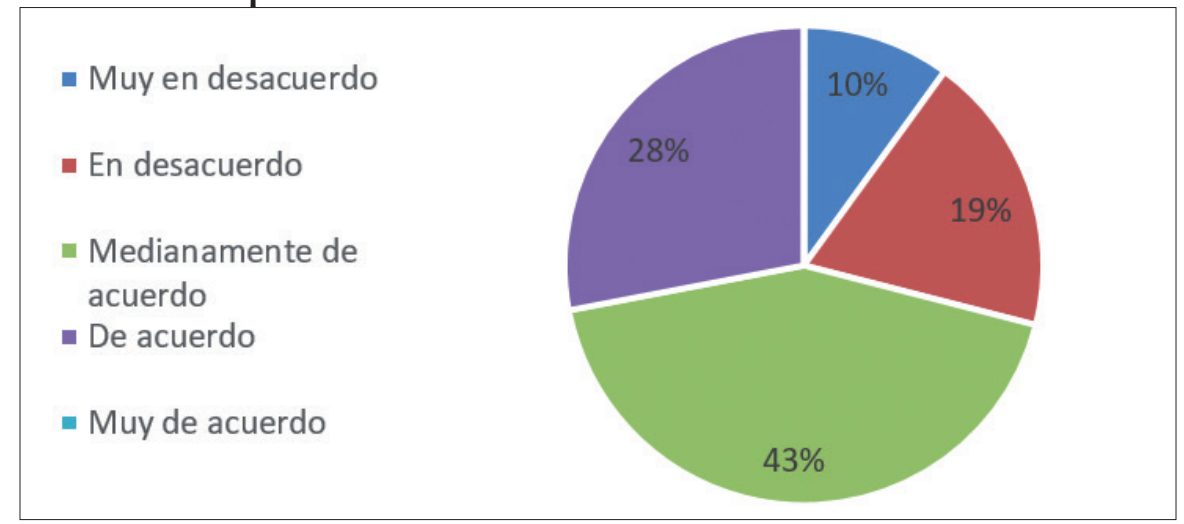

planificadas con metodología de tutoría.

Un 43\% muestran estar "medianamente de acuerdo", puesto que algunos consideran muy poco el tiempo de tutoría y de preparación del seminario. Sin embargo hay un $28 \%$ que indica estar "de acuerdo" en el número de tutorías.

El análisis se detiene en la Dimensión 4: Relación docente-estudiantes y se hallan los resultados siguientes: 
- "El docente mostró buena disposición a responder las consultas"
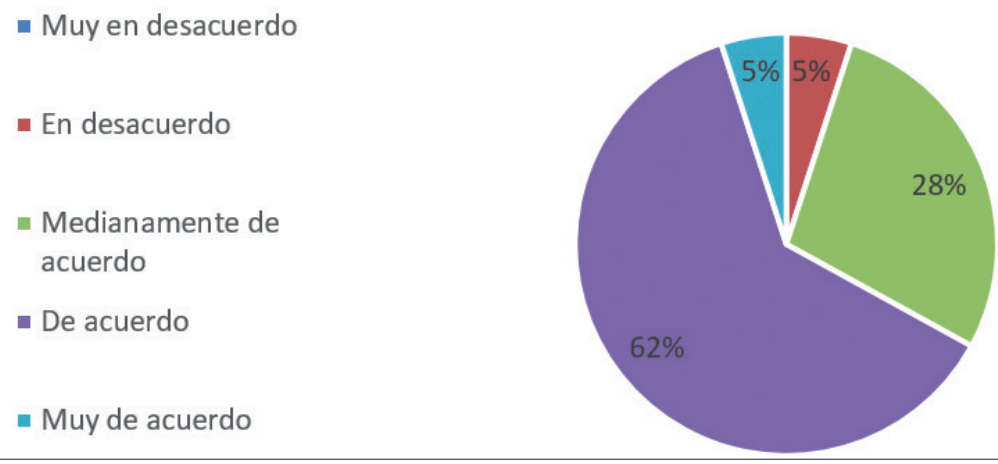

Gráfico 7. Respuestas de los estudiantes en relación a la disposición del docente por responder a las consultas de los estudiantes.

Más de la mitad de los participantes, el 62\% manifiesta estar "de acuerdo" en que el docente muestra una buena disposición para guiarlos en sus consultas y aclarar dudas, generando motivación en los estudiantes. Un 28\% manifiesta estar "medianamente de acuerdo" y sólo un $5 \%$ de los encuestado indica estar "en desacuerdo", lo que refleja que el académico en general responde a las consultas de los estudiantes.

- "El docente proporciona feedback positivo a los estudiantes, refuerza las intervenciones, escucha al estudiante atento"

- Muy en desacuerdo

- En desacuerdo

- Medianamente de acuerdo

- De acuerdo

- Muy de acuerdo

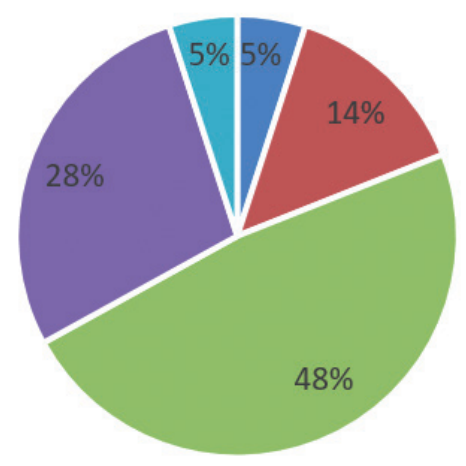

Gráfico 8. Respuestas de los estudiantes en relación a la entrega de feedback positivo por parte del docente. 
El 48\% declara estar "medianamente de acuerdo" en que el docente entrega refuerzo positivo a los estudiantes, ya que si bien responde a las preguntas, difícilmente entrega mayor refuerzo positivo; un 28\% manifiesta estar "de acuerdo", por lo que se observa que hay estudiantes que si se motivan con las respuestas del docente. Sin embargo un $14 \%$ de los estudiantes encuestado indica estar "en desacuerdo", por lo que hay que analizar la estrategia que usa el docente para incentivar a los estudiantes a desarrollar las actividades y aprender a aprender.

Igualmente, respecto a Sección II: Evaluación global, se usa la misma escala de evaluación que para la sección anterior y se obtienen estos resultados:

- "Recomendaría la aplicación de esta metodología en otras asignaturas de la carrera de Ingeniería Ambiental"

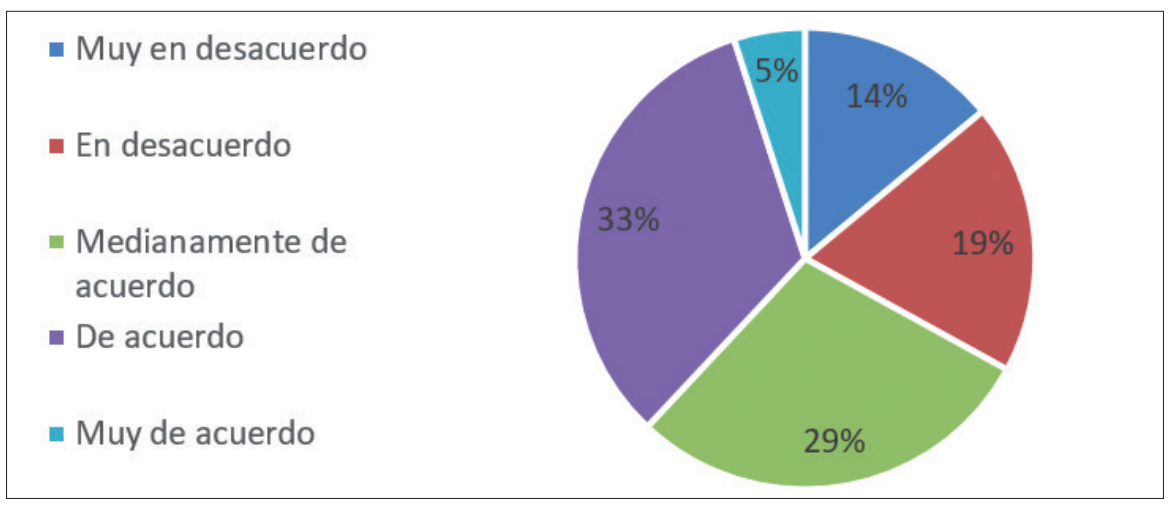

\section{Gráfico 9. Respuesta de los estudiantes en relación a la recomendación de esta metodología para otras asignaturas de la malla curricular.}

El 33\% exhiben estar "de acuerdo" en que recomendarían la aplicación de esta metodología en otras asignaturas de la carrera, puesto que sienten que les agrada y les parece que es una buena oportunidad para aprender; un 29\% manifiesta estar "medianamente de acuerdo" con la afirmación y un 19\% de los encuestado indica estar "en desacuerdo" ya que indican que con la metodología no logran el aprendizaje esperado, por lo que hay que analizar si la aplicación de la metodología fue la adecuada para los estudiantes, y si éstos están preparados, puesto que llevan varios semestres aprendiendo con metodología tradicional en forma pasiva. 
- ¿Qué calificación le pondría a la metodología tradicional de carácter expositivo?

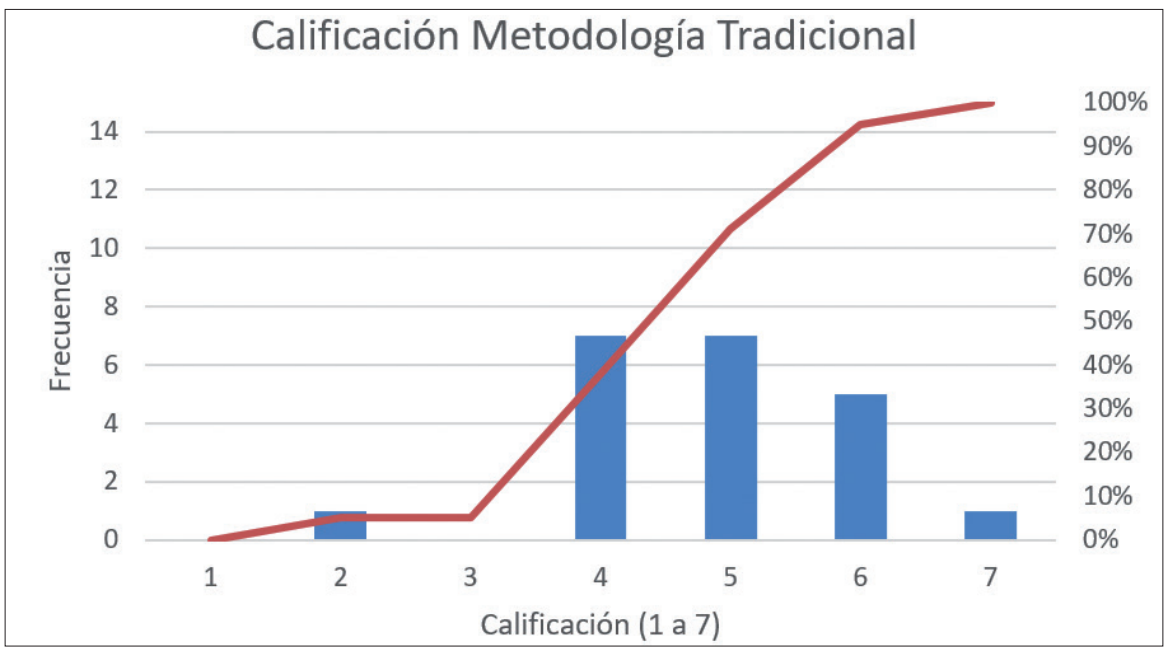

Grafico 10. Calificación de los estudiantes a la metodología tradicional.

El 33\% de los estudiantes encuestados calificó con nota 4,0 y un 33\% con 5,0 la metodología tradicional expositiva. El promedio general obtenido fue de un 4,5, lo que indica que si bien les agrada la metodología tradicional, no es tan valorada.

- ¿Qué calificación le pondría a la metodología de tutoría empleada en la unidad 2 de esta asignatura?

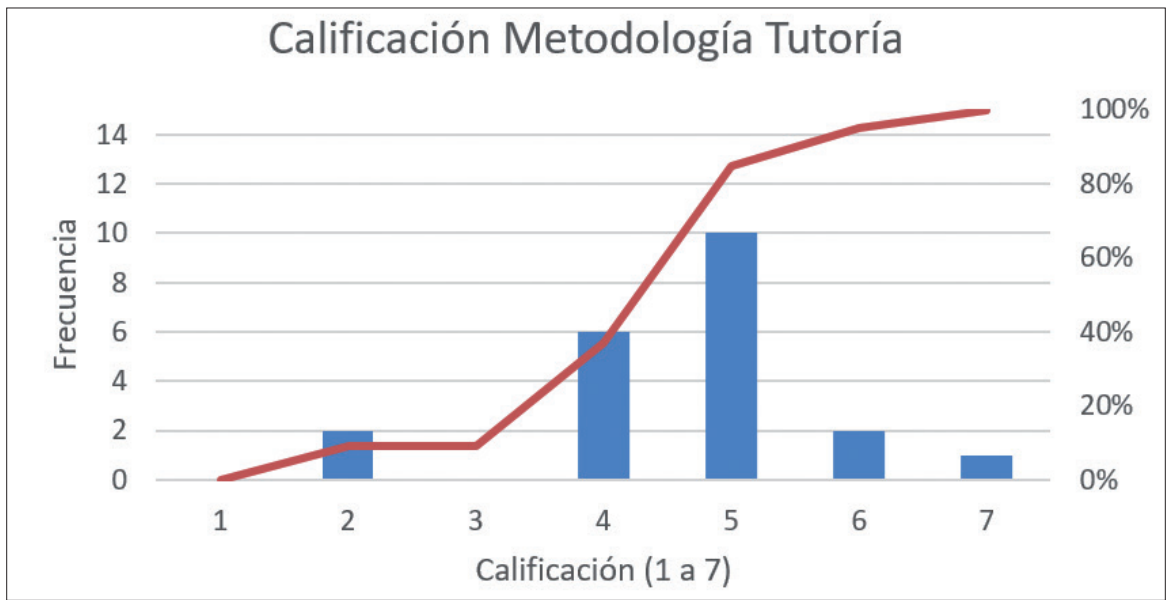

Gráfico 11. Calificación de los estudiantes a la metodología de tutoría. 
El 48\% de los encuestados evalúa la metodología de tutoría con un 5,0 el 29\% la evalúa con un 4,0. El promedio es de un 4,6, lo que indica que si bien, la metodología es valorada, existe desconfianza de su efectividad, puesto que el estudiante debe ser más autónomo y tener un rol más protagonista al que tal vez no está acostumbrado. Al parecer los estudiantes confunden la metodología de estudio de caso con la resolución de problemas, lo que podría incidir en su percepción de la metodología. También tienen reparos en el tiempo dispuesto para las tutorías, ya que, al no tener clases expositivas, sienten que el docente no les está entregando los conocimientos necesarios, lo que demuestra nuevamente la poca experiencia en tener un rol más activo, ya que esperaban que el docente finalmente les diera la respuesta.

Respecto al Objetivo 3 se realizó un análisis valorativo de los comentarios y sugerencias propuestas por los participantes. Se registraron 16 respuestas. Los temas con mayor número de menciones tienen relación con "metodología", "retroalimentación" y "actitud del docente". A continuación se exponen los comentarios registrados:

- La metodología de Tutoría a mi parecer es buena, da resultados positivos en el aprendizaje de los alumnos, sin embargo considero que no tiene el mismo resultado en todas las asignaturas, de acuerdo a ellos, contaminación atmosférica que requiere de cálculos y no tanta teoría, podría enfocarse en lo expositivo más que en tutorías (sólo por efectos de cálculo)

- Me parece interesante la metodología de resolución de problemas que usa el profesor, ya que nos da la oportunidad de aplicar conocimientos teóricos adquiridos previamente en otras asignaturas e incentiva la curiosidad y la capacidad de análisis de los estudiantes.

- En mi opinión una metodología de enseñanza de tutoría es una muy buena oportunidad de aprendizaje, pero considero necesario tener una o al menos dos clases de carácter expositivo, para poder contextualizar las problemáticas a nivel de resolución de problemas.

- La metodología en sí no es una mala práctica, pero se tendría que considerar un mayor tiempo de disponibilidad de parte del docente para el desarrollo del trabajo final.

- El método de enseñanza es bueno, el docente podría hacer una mayor crítica porque esto no se refleja en sus evaluaciones.

- Si bien podemos pasar de un estado A, a un estado B y aprender 
Un caso de tutoría grupal: aplicación de técnicas de aprendizaje cooperativo orientado a resolución de problemas de la especialidad en ingeniería ambiental

María Paz Araya Cabrera, Héctor Andrés Andrade Caroca y Joao Ricardo Cerqueira Pinto

bastantes cosas que algunos aprendieron en el pasado y otros no, es un método poco ortodoxo y sacrificado, pero es funcional, se sufre pero se aprende.

- El método utilizado en sí puede ser bastante "llenador" para un estudiante, ya que no es tan metódico como el tradicional. Sin embargo, el docente claramente presenta actitudes que llevan a que el estudiante pueda "frustrarse" al ver situaciones en que no se sabe cuáles son los errores que uno comete.

- Es una buena idea pero toma más tiempo que la otra metodología, por lo tanto, si se implementara en otros ramos no sé cómo se podrían organizar loa tiempos de los estudiantes.

- La metodología de tutoría, si bien hace que los estudiantes busquen información necesaria por su cuenta, deja en abandono la enseñanza de la aplicación clave, además de la entrega de herramientas que se utilizarán en la asignatura. Además de no realizar la corrección del trabajo para evaluar si lo hicimos bien o mal, dejándonos con un vacío de aprendizaje muy amplio, los cuales se mantienen en la siguiente unidad.

- El tipo de enseñanza (tutoría) con el fin de un mejor desarrollo, se sugiere realizar más sesiones para aclaraciones y feedback.

- Si bien el profesor chequea el avance de los estudiantes, éste no da abasto para todos, puesto que no pudo atender a todos los grupos en los dos días habilitados que tenía a la semana. Me gusta la metodología, pero al final del trabajo no estoy segura de haber aprendido correctamente. Se debe revisar el trabajo.

- Cuando se haga este tipo de metodología, sería muy bueno que se generaran instancias correctivas antes de una evaluación, ya que si bien el método es bueno, requiere de mucha dedicación y tiempo, por lo cual recibir malas calificaciones es contraproducente para el estudiante, tomando en cuenta todo el tiempo y trabajo invertido.

- Considero personalmente que se deberían realizar más ejercicios en clases, desarrollándolos y explicándolos completamente, porque cuando se parte de no saber nada se pierde mucho tiempo intentando hacer un ejercicio que ni tengo la seguridad de estar haciendo bien.

- Que el profesor sea más claro en lo que solicita y que el profesor no dé por sabido materia que no recordamos.

- Siento que el profesor, o se observa que el profesor siempre tuvo mejor disposición cuando estaba la persona evaluadora en la mis- 
ma sala, su personalidad cambiaba al igual que su disposición hacia los alumnos cuando no estaba.

- El profesor con sus comentarios me hace ver insignificante y que no debo seguir en la carrera, además no estoy de acuerdo en el tipo de preguntas que realiza en las pruebas, no las encuentro acorde al ramo.

Estos comentarios indican que en general los estudiantes perciben que la metodología implementada es efectiva pero, hay que ir afinando detalles y generar más material de apoyo, que es lo natural que va a ocurrir al generarse la necesidad. La retroalimentación es uno de los puntos débiles que hay que reforzar para que los estudiantes tengan mayor certeza de los aprendizajes logrados. Se sienten bien con el trabajo colaborativo, pero son susceptibles a los comentarios negativos del docente cuando los estudiantes no cumplen con sus expectativas, lo que genera en algunos casos un clima desfavorable para el aprendizaje.

En lo relativo al Objetivo 4, podemos extraer que al comparar las cuatro dimensiones encuestadas, la dimensión más valorada fue "Organización estructural de la sesión o cátedra" con las afirmaciones: "Los contenidos de la sesión me han permitido integrar/ relacionar/ comparar conocimientos con otras asignaturas y/o con situaciones de la vida real" y "Tuve oportunidad de desarrollar/ resolver casos o problemas reales", lo que evidencia que la metodología de estudio de casos y tutoría fue estimada ya que hubo instancias en que el estudiante podía aplicar conocimientos previos en un contexto real de la disciplina e integrarlo con nuevos conocimientos. Dentro de ésta dimensión la afirmación menos valorada fue: "Me han quedado claros los objetivos que se establecieron para las sesiones", lo que indica que el estudiante no tiene claridad o no valora aún el real aporte de la asignatura a su perfil profesional.

La segunda dimensión "La Actitud del Estudiante frente a la clase" fue una de las menos valorada con las afirmaciones: "El método de tutoría contribuye significativamente al logro de mis aprendizajes" y "Si me dan a elegir, prefiero este tipo de enseñanza", en donde sólo están medianamente de acuerdo con estas afirmaciones, lo que refleja que los estudiantes si bien valoran la metodología, no están acostumbrados a tener un rol más protagonista. La afirmación más valorada fue "El método de tutoría contribuye significativamente a integrar conocimientos de asignaturas anteriores", lo que indica que el estudiante reconoce la integración de conocimientos previos con los nuevos conocimientos. 
La tercera dimensión "Estrategias de seguimiento y evaluación" fue bien apreciada, durante las sesiones existieron instancias donde el docente podía chequear el avance de los estudiantes (preguntas, autoevaluación, coevaluación, otros) y el estudiante las reconoce. La afirmación menos valorada fue "Considera adecuado el número de sesiones planificadas para el desarrollo de la tutoría (una para la presentación, cinco para aclaraciones y feedback y dos para la presentación de los resultados)" ya que los estudiantes perciben que no fueron suficientes las instancias de tutoría ni el tiempo de preparación del seminario ya que el tema de buscar información por su cuenta les es complejo, lo que refleja su debilidad en investigación autónoma.

En relación a la cuarta dimensión "Relación docente-estudiante" también fue una de las más valoradas la afirmación: "El docente mostró buena disposición a responder las consultas", lo que se reflejó bastante en las sesiones de tutoría. Sin embargo la afirmación menos valorada fue: "El docente proporciona feedback positivo a los estudiantes, refuerza las intervenciones, escucha al estudiante atento", ya que algunos estudiantes perciben que el docente no los entusiasma, sino al contrario al hacer comentarios negativos más que positivos.

En relación a la evaluación global, esta se considera positiva puesto que su evaluación se acerca a lo óptimo, por lo que se entiende que la aplicación de ésta metodología activa es perfectible en el tiempo, si se toman en consideración algunas de las sugerencias de la experiencia.

Finalmente, para el Objetivo 5: Identificar el impacto de la aplicación de la tutoría en los resultados académicos de los estudiantes, se han hallado los estadísticos brutos para los últimos años siendo estos los siguientes:

Tabla 1. Estadísticos brutos para los últimos años.

\begin{tabular}{ccccc}
\hline Año & $\mathbf{2 0 1 3}$ & $\mathbf{2 0 1 4}$ & $\mathbf{2 0 1 5}$ & $\mathbf{2 0 1 6}$ \\
\hline Número & 27 & 21 & 31 & 23 \\
Máximo & 4,9 & 4,6 & 5,3 & 5,1 \\
Mínimo & 2,3 & 2,6 & 1,0 & 2,0 \\
Promedio & 3,7 & 3,9 & 3,8 & 3,6 \\
Desviación Estándar & 0,66 & 0,40 & 0,74 & 0,86 \\
Mediana & 4,0 & 4,0 & 4,0 & 3,4 \\
Percentil 75 & 4,0 & 4,0 & 4,1 & 4,2 \\
Percentil 25 & 3,3 & 3,8 & 3,6 & 3,0 \\
Tasa Aprobación & 63 & 67 & 68 & 48 \\
\hline
\end{tabular}


Suponiendo que la única diferencia entre los años anteriores y el actual fue la aplicación de la Tutoría, es posible concluir de los descriptores que fue negativo desde el punto de vista de la tasa de aprobación, ya que se pasó de una tasa cercana al $70 \%$ a una inferior al $50 \%$, esto debido a que ocho estudiantes no finalizaron la tutoría, ya que no entregaron el trabajo final.

En caso de eliminar aquellos estudiantes que no finalizaron la tutoría y que fueron calificados con nota mínima, los descriptores estadísticos quedan de la siguiente forma:

Tabla 2. Descriptores estadísticos.

\begin{tabular}{ccccc}
\hline Año & $\mathbf{2 0 1 3}$ & $\mathbf{2 0 1 4}$ & $\mathbf{2 0 1 5}$ & $\mathbf{2 0 1 6}^{\prime}$ \\
\hline Número & 27 & 21 & 31 & 15 \\
Máximo & 4,9 & 4,6 & 5,3 & 5,1 \\
Mínimo & 2,3 & 2,6 & 1,0 & 2,7 \\
Promedio & 3,7 & 3,9 & 3,8 & 3,8 \\
Desviación Estándar & 0,66 & 0,40 & 0,74 & 0,85 \\
Mediana & 4,0 & 4,0 & 4,0 & 4,0 \\
Percentil 75 & 4,0 & 4,0 & 4,1 & 4,4 \\
Percentil 25 & 3,3 & 3,8 & 3,6 & 3,2 \\
Tasa Aprobación & 63 & 67 & 68 & 73 \\
\hline
\end{tabular}

De lo anterior, se ve una mejora significativa en la tasa de aprobación y los descriptores restantes también presentan mejoras.

Como último elemento de análisis, se considera la correspondencia entre los ocho (8) estudiantes que no entregaron el trabajo correspondiente a la Tutoría y su tasa de aprobación, la que fue cercana a un 38\% (3 de 8 aprobaron la asignatura). Lo anterior podría interpretarse con el desarrollo insuficiente de habilidades blandas como la autorregulación, ya que habría una importante diferencia en la tasa de aprobación entre aquellos estudiantes que presentaron el trabajo final y aquellos que no lo presentaron.

\section{Conclusiones y Discusión}

Como principales conclusiones del estudio realizado, se pueden extraer las siguientes: Los estudiantes manifiestan tener problemas para relacio- 
nar conocimientos de asignaturas anteriores con las actuales lo que demuestra que las metodologías usadas en las anteriores asignaturas son básicamente conductistas con memorización de contenidos y poco o ineficiente uso de metodologías activas, lo que hace que el estudiante olvide prontamente los conocimientos adquiridos en las sesiones.

Los estudiantes carecen de estrategias de búsqueda de información, lo que se manifiesta en las sesiones de tutoría en donde la mayoría de los estudiantes indica que no sabe dónde buscar información técnica específica solicitada por el docente.

La tutoría universitaria se concibe como un proceso orientador de carácter formativo que facilita este desarrollo y formación integral en su proyección académica, profesional y personal. Se trata de acompañar al estudiante a lo largo de su aprendizaje, con la intención de potenciar y favorecer su proyecto profesional y de vida. Desde esta concepción la tutoría universitaria se construye más allá de la mera resolución de problemas puntuales y concretos, ya que comporta una relación de apoyo y ayuda entre profesor-tutor y alumno, que debe hacerse extensiva a toda la dinámica que conforma el proceso educativo, como también señala el estudio de Martínez Clares, P., Pérez Cusó, J. y Martínez Juárez, M., (2014)

Debemos abandonar la concepción puramente burocrática de la tutoría por otra más amplia, más comprensiva, que vaya más allá del quehacer de todo profesor universitario en el desarrollo de sus asignaturas y que abarque aspectos relacionados con la madurez y el desarrollo integral del alumnado. Defendemos por tanto "una tutoría cuyo ámbito de actuación no se restringe puramente [...] al horario de las 6 horas y al espacio de la oficina [...], sino que se extiende más allá, haciendo un seguimiento del proceso formativo y estimulando la madurez personal y profesional, con una clara proyección hacia el aprendizaje autónomo, la investigación y el desempeño profesional", tal y como señala Álvarez Pérez (2005, p.284). Esta redefinición de la tutoría conlleva nuevas responsabilidades y modos de hacer que exigen la implicación de la institución universitaria a todos sus niveles.

En la actualidad, la tutoría universitaria resulta singularmente apropiada y necesaria para dar respuesta a las demandas del nuevo modelo universitario. El resultado, una vez Ilevado al aula este modelo, es positivo en varios aspectos ya que se desarrollan competencias de tipo instrumental, interpersonal y sistémico reflejado por ejemplo en el au- 
Un caso de tutoría grupal: aplicación de técnicas de aprendizaje cooperativo orientado a resolución de problemas de la especialidad en ingeniería ambiental María Paz Araya Cabrera, Héctor Andrés Andrade Caroca y joao Ricardo Cerqueira Pinto

mento de la participación en clases. Sin embargo también se presentan situaciones desfavorables ya que se evidencia el desconocimiento del método por parte de los docentes de la escuela y la poca disposición de los estudiantes a desarrollar un trabajo más autónomo.

\section{Referencias bibliográficas}

Álvarez Pérez, P. (2005). La tutoría y la orientación universitaria en la nueva coyuntura de la enseñanza superior: El Programa "Velero". Revista Contextos Educativos (Revista de Educación), 8, 281-293.

Buendía, L., Colás, P., \& Hernández, F. (1998). Métodos de Investigación en Psicopedagogía. Madrid: McGraw Hill.

González Maura, V., \& González Tirados, R. M. (2007). Competencias genéricas y formación profesional: un análisis desde la docencia universitaria. Revista Iberoamericana de Educación, 47, 185-209.

Hernández, M. E., \& Domínguez, D. (1998). La nueva acción tutorial en la enseñanza universitaria. Revista de Enseñanza Universitaria, 13, 79-88.

Lázaro, A. (1997). La acción tutorial de la función docente universitaria. Revista Complutense de Educación, 8(1), 233-252.

Martínez Clares, P., Pérez Cusó, J. y Martínez Juárez, M. (2014). Una (re)visión de la tutoría universitaria: la percepción de estudiantes y tutores de estudios de Grado. Revista de Docencia Universitaria, 12 (1), 269-305.

Martínez, M., Buxarrais, M. R., \& Esteban, F. (2002). Ética y formación universitaria. Revista Iberoamericana de Educación, 29, 17-43.

Mesa, V. (2010). Student Participation in Mathematics Lessons Taughtby Seven Successful Community College Instructors. Ann Arbor, MI University of Michigan. ALM International Journal,Vol.5 (1), 64-88.

Pino, M. R., \& Ricoy, M. C. (2006). La tutoría como recurso en la enseñanza universitaria. Estudio de necesidades y contribuciones actuales. Revista Bordón, 58 (3), 345-358.

Rodríguez Moreno, M. L. (2002). Hacia una nueva orientación universitaria: Modelos integrados de acción tutorial, orientación curricular y construcción del proyecto profesional. Barcelona: EUB.

Zabalza, M. A. (2003). Competencias docentes del profesorado universitario: Calidad y desarrollo profesional. Madrid: Narcea. 
